\title{
Theoretical and experimental investigation of sound power transmitting through reflecting plane with low surface density in the calibration of reference sound sources
}

\author{
Keisuke Yamada*, Hironobu Takahashi ${ }^{\dagger}$ and Ryuzo Horiuchi ${ }^{\ddagger}$ \\ National Metrology Institute of Japan/National Institute of Advanced Industrial Science and Technology, \\ 1-1-1 Umezono, Tsukuba, 305-8563 Japan
}

(Received 1 December 2014, Accepted for publication 20 January 2015)

Keywords: Reference sound source, Sound power level, Transmission coefficient, Mass law, Surface density, Free-field over a reflecting plane PACS number: 43.58.Vb [doi:10.1250/ast.36.374]

\section{Introduction}

The sound power level is used for quantitative evaluation of sound energy radiated from electrical and mechanical apparatus. Procedures for measuring the sound power level are categorized into the ISO 3740 series (such as ISO 3745 [1]) on the basis of measurement principle, environment, and accuracy. Among them, practical procedures involve the use of a reference sound source (RSS) with a predetermined sound power level, and an apparatus under test can be easily calibrated in comparison with the RSS. The RSS has stable and broad-band sound power output. Requirements for its performance and calibration procedure are prescribed in ISO 6926 [2]. Thus precise calibration of the RSS is essential for end-users to establish reliable sound power measurement.

In ISO 6926, the RSS is calibrated in a hemi-anechoic or reverberation room. From the point of accuracy, we decided to use a hemi-anechoic room. However, we have access to only an anechoic room, but a hemi-free-field is realized by laying down wooden boards on the wire-meshed floor of the anechoic room. It is difficult to lay down boards with high surface density owing to the load capacity of the wire-meshed floor, even though ISO 3745 requires that the absorption coefficient of the reflecting plane should be less than 0.06 for the frequency range of interest.

A previous study on calibration of the RSS revealed that there is no significant difference between a solid concrete and a lightweight wooden floor when determining the sound power level [3]. However, no detailed description has been found of the surface density of the floor and the influence of the surface density on the sound power level. Thus, we investigate sound power transmitting through the wooden board floor quantitatively.

In this work, we formulate the sound power transmitting through the floor, using the mass law. By using the derived formulae, we examine the dependence of the transmitted sound power on the surface density of the floor. Then experimental results obtained with our calibration system are compared with theoretical values.

\footnotetext{
*e-mail: keisuke.yamada@aist.go.jp

†e-mail: h.takahashi@aist.go.jp

‡e-mail: ryuzo.horiuchi@aist.go.jp
}

\section{Calibration system}

Figure 1 shows the schematic of our calibration system to determine the sound power level of the RSS in accordance with ISO 6926. The RSS used is Brüel \& Kjær 4202 because it is a de facto standard. The reflecting plane consists of wooden boards each of which is $0.92 \mathrm{~m} \times 1.83 \mathrm{~m} \times 24 \mathrm{~mm}$ in size. Wooden boards are double-stacked and the surface density is $15.0 \mathrm{~kg} / \mathrm{m}^{2}$. The reflecting plane is $26.9 \mathrm{~m}^{2}$ in area and covers 35 percent of the wire-meshed floor. The dimensions of the space within the anechoic room are $9.5 \mathrm{~m}$ in width, $8.0 \mathrm{~m}$ in depth and $7.2 \mathrm{~m}$ in height [4]. The wiremeshed floor is located $5.6 \mathrm{~m}$ below the ceiling of the room. The cut-off frequency of sound-absorbing wedges is $40 \mathrm{~Hz}$.

The sound power level of the RSS is determined from the surface sound pressure level. We designed the hemispherical frame to realize a measurement surface with the radius of $2.0 \mathrm{~m}$ [5]. The RSS is set on the floor at the center of the frame. Twenty WS2F microphones [6] (MI-1234, Onosokki) are fixed to the frame using microphone holders and connected to preamplifiers (MI-3111, Onosokki). The output voltage of each microphone is acquired simultaneously by a $20 \mathrm{ch}$ FFT analyzer (DS-2000, Onosokki). A PC automatically controls the FFT analyzer and calculates the sound power level.

\section{Formulation of sound power transmitting through floor}

We theoretically investigate the sound power transmitting through the floor in our system. The RSS is modeled as a point source located at the geometric center of the RSS. The RSS and floor are depicted in Fig. 2.

$S$ denotes the projection of the hemispherical measurement surface on the floor, with radius $r$ of $2.0 \mathrm{~m}$, as described above. $d$ is the distance between the geometric center of the RSS and the floor. $W_{\text {trans }}$ is the sound power transmitting through $S$ and is expressed as

$$
W_{\text {trans }}=\int_{S} \tau_{\theta} \boldsymbol{I} \cdot \boldsymbol{n} d S,
$$

where $\tau_{\theta}$ is the transmission coefficient of sound power for sound with incident angle $\theta, \boldsymbol{I}$ is the sound intensity, and $\boldsymbol{n}$ is the unit vector normal to $S$. We assumed that sound power is not absorbed within the floor, considering the thickness of the floor. 


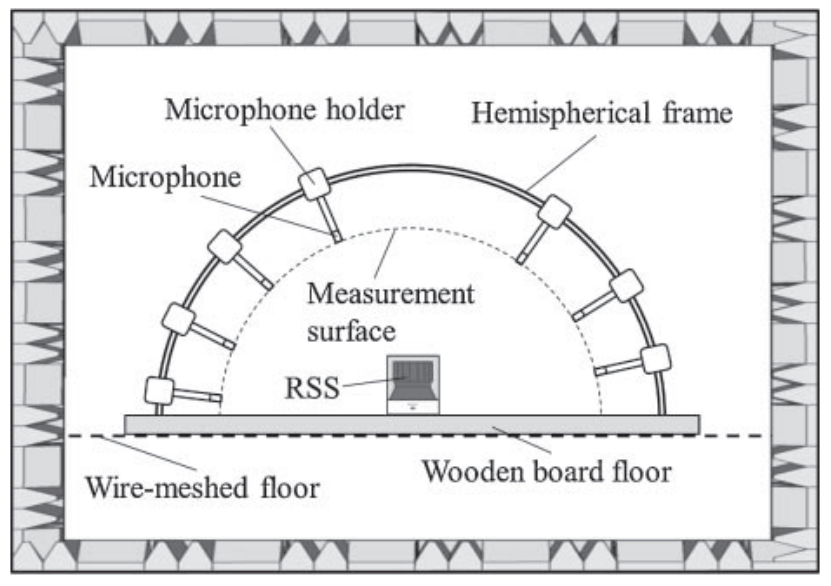

Fig. 1 Schematic of developed calibration system for sound power level of RSS.

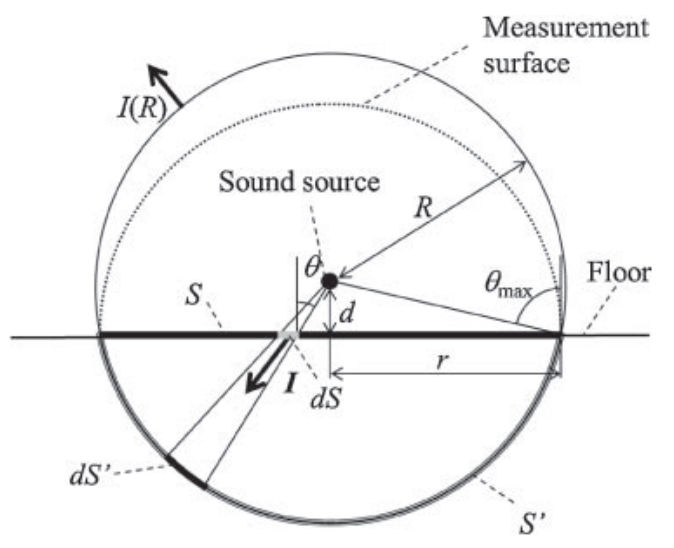

Fig. 2 Schematic of sound source and reflecting plane in our calibration system.

Equation (1) can be rewritten by introducing polar coordinates with the origin at the position of the point source. As shown in Fig. 2, the area $S$ corresponds to $S^{\prime}$ on the spherical surface of radius $R$ and sound intensity magnitude $I(R)$ is written as

$$
I(R)=\frac{W_{0}}{4 \pi R^{2}},
$$

where $W_{0}$ is the total sound power radiated from the point source. The area element $d S^{\prime}$ in area $S^{\prime}$ is written as

$$
d S^{\prime}=R^{2} \sin \theta d \varphi d \theta,
$$

where $\varphi$ is the azimuthal angle in polar coordinates.

Thus Eq. (1) is changed to

$$
\begin{aligned}
W_{\text {trans }} & =\int_{0}^{\theta_{\max }} \int_{0}^{2 \pi} \tau_{\theta} \frac{W_{0}}{4 \pi R^{2}} R^{2} \sin \theta d \varphi d \theta \\
& =\frac{W_{0}}{2} \int_{0}^{\theta_{\max }} \tau_{\theta} \sin \theta d \theta
\end{aligned}
$$

where $\theta_{\max }$ is uniquely defined by $r$ and $d$ as $\tan ^{-1}(r / d)$ for the same type of RSS. Equation (4) also implies that $W_{\text {trans }}$ does not depend on $R$. Thus we do not discuss the dependence of $W_{\text {trans }}$ on $R$ and $\theta_{\text {max }}$.
From the mass law [7], the transmission coefficient $\tau_{\theta}$ for a plane wave of incident angle $\theta$ is given by

$$
\tau_{\theta}=\frac{1}{1+\left(\frac{\omega m}{2 \rho c} \cos \theta\right)^{2}},
$$

where $\omega$ is the angular frequency of the sound, $m$ is the surface density of the floor, $\rho$ is the density of air and $c$ is the sound speed in air.

From Eqs. (4) and (5), we derive the formulae of sound power transmitting through the floor as a function of surface density of the floor:

$$
\frac{W_{\text {trans }}(m)}{W_{0}}=\frac{1}{2} \int_{0}^{\theta_{\max }} \frac{1}{1+\left(\frac{\omega m}{2 \rho c} \cos \theta\right)^{2}} \sin \theta d \theta .
$$

The sound power to be observed at the hemispherical measurement surface is expressed as

$$
\left.W_{\text {measure }}\right|_{m}=W_{0}-W_{\text {trans }}(m) .
$$

Finally, the following formula is derived.

$$
\begin{aligned}
L_{W_{-} \text {ratio }}(m) & =-10 \log _{10}\left(\frac{\left.W_{\text {measure }}\right|_{m}}{W_{0}}\right) \\
& =-10 \log _{10}\left(1-\frac{W_{\text {trans }}(m)}{W_{0}}\right)
\end{aligned}
$$

$L_{W_{\text {_ratio }}}$ represents the ratio of the sound power observed at the measurement surface relative to the total sound power radiated from the point source. The sign of $L_{W_{-} \text {ratio }}$ is changed for convenience.

\section{Dependence of transmitting sound power on surface density of floor}

Using Eq. (8), we quantitatively examine the dependence of transmitting sound power on the surface density of the floor. The examination is carried out for the surface densities of $5.0,7.5,15.0$, and $20.0 \mathrm{~kg} / \mathrm{m}^{2}$. The surface densities of 7.5 and $15.0 \mathrm{~kg} / \mathrm{m}^{2}$ correspond to the single-stacked and doublestacked wooden boards in our system, respectively. Air density and sound speed are $1.2 \mathrm{~kg} / \mathrm{m}^{3}$ and $340 \mathrm{~m} / \mathrm{s}$. The distance $d$ from the geometric center of the RSS to the floor is $16 \mathrm{~cm}$ and $\theta_{\max }$ is $85.4^{\circ}$ for the Brüel \& Kjær $4202 \mathrm{RSS}$. The frequency of interest ranges from $100 \mathrm{~Hz}$ to $10 \mathrm{kHz}$ for the center frequency of the $1 / 3$ octave band, in accordance with ISO 6926.

Figure 3 shows the theoretical relationship between $L_{W_{\text {_ratio }}}$ and the surface density of the floor. At frequencies less than or equal to $1 \mathrm{kHz}, L_{W_{-} \text {ratio }}$ increases as frequency decreases. $L_{W \text { _ratio }}$ also increases as surface density decreases. On the other hand, at frequencies over $1 \mathrm{kHz}, L_{W_{\text {_ratio }}}$ is about $0.01 \mathrm{~dB}$, which is negligible, independent of surface density and frequency.

\section{Experimental results}

Using Eq. (8), we experimentally examine the dependence of the transmitting sound power on the surface density of the floor. However, practically, the only quantity we can measure is $\left.W_{\text {measure }}\right|_{m}$ and $W_{0}$ cannot be experimentally obtained. To cancel out the influence of $W_{0}$, we performed 


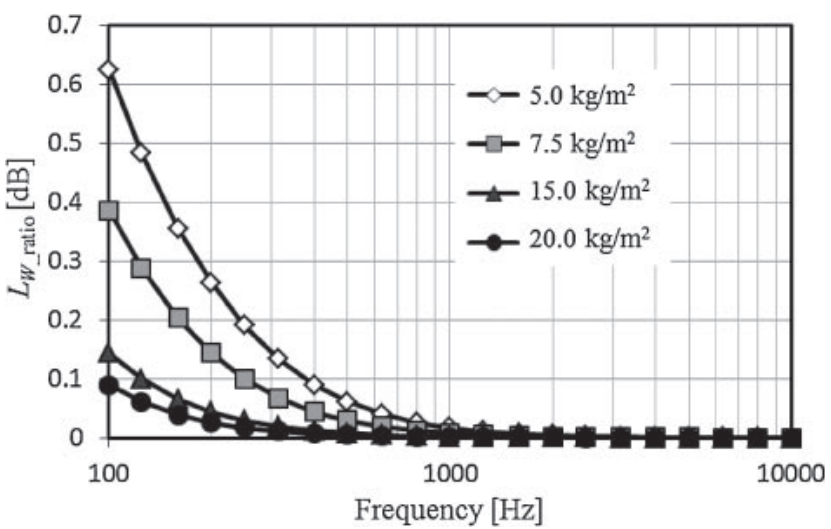

Fig. 3 Theoretical calculation of $L_{W_{-} \text {ratio }}$ for surface densities of $5.0,7.5,15.0$, and $20.0 \mathrm{~kg} / \mathrm{m}^{2}$.

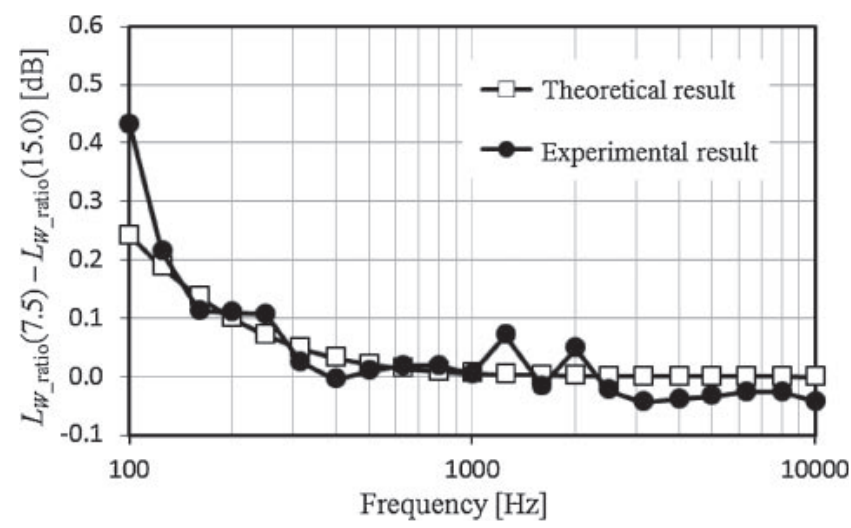

Fig. 4 Experimental and theoretical results of $L_{W_{\text {ratio }}}$ (7.5) relative to $L_{W_{\text {_ratio }}}(15.0)$.

two kinds of measurement for the surface density, $m_{1}$ and $m_{2}$. We tried to verify Eq. (8) by using the relative difference between $L_{W_{-} \text {ratio }}\left(m_{1}\right)$ and $L_{W_{-} \text {ratio }}\left(m_{2}\right)$.

Experimental conditions are as follows: Brüel \& Kjær 4202 RSS driven by AC $100 \mathrm{~V}$ with $50 \mathrm{~Hz}$, surface density $m_{1}$ of $15.0 \mathrm{~kg} / \mathrm{m}^{2}$, and $m_{2}$ of $7.5 \mathrm{~kg} / \mathrm{m}^{2}$. For each surface density, sound power was measured three times at intervals of 15 minutes and averaged. The standard deviation of the sound power was less than $0.04 \mathrm{~dB}$ for all frequencies. The environmental conditions during measurement were $23.3 \pm 0.2^{\circ} \mathrm{C}$ temperature and $100.0 \pm 0.1 \mathrm{kPa}$ static pressure.

Figure 4 shows that experimental results are mostly in good agreement with theoretical values within $0.1 \mathrm{~dB}$, except at $100 \mathrm{~Hz}$ where the difference between theoretical and experimental results is $0.2 \mathrm{~dB}$.

\section{Discussion}

Figures 3 and 4 show the following findings. At frequencies over $1 \mathrm{kHz}$, Fig. 3 shows that, theoretically, the transmitting sound power is negligible for the surface density of the wooden board floor in our calibration system and the experimented result in Fig. 4 validated this hypothesis. Thus above $1 \mathrm{kHz}$, the sound power level determined in our system is not influenced by the surface density of the floor.

From $125 \mathrm{~Hz}$ to $1 \mathrm{kHz}$, Fig. 4 showed that experimental results are in good agreement with theoretical values and that the transmitting sound power does increase as the surface density of the floor decreases. This means that the transmitting sound power can be theoretically estimated and that the sound power measured in our system can be corrected for precise sound power calibration.

At $100 \mathrm{~Hz}$, Fig. 4 showed that there is an unexpected discrepancy of $0.2 \mathrm{~dB}$ between the experimental and theoretical results. One possible reason for this discrepancy might be the assumption of the mass law in deriving Eq. (5); the mass law assumes an infinite surface, whereas the actual floor is finite. At frequencies less than or equal to $100 \mathrm{~Hz}$, however, the wavelength of the sound becomes longer than the distance between the RSS and the floor edge. Sound diffraction at the floor edge might cause the discrepancy between the experimental and theoretical results. Further study is needed to determine the sound power transmission at $100 \mathrm{~Hz}$ more accurately, but this discrepancy is practically acceptable.

Furthermore, we examined the influence of floor vibration on the sound power level [5]. The RSS is physically isolated from the floor by hanging it from the ceiling of the anechoic room using fine steel wires. The surface density of the floor is $15.0 \mathrm{~kg} / \mathrm{m}^{2}$. We concluded that the influence of floor vibration is less than $0.1 \mathrm{~dB}$ for all frequencies. This means that the influence of floor vibration is not significant in our system.

\section{Conclusion}

The sound power transmitting through the floor was formulated using the mass law and verified by experiment. The wooden board floor in our system is not acoustically rigid but it was confirmed by experiment that the sound power transmission can be theoretically corrected.

\section{References}

[1] ISO 3745: 2003 (JIS Z 8732: 2000), Determination of sound power levels of noise sources using sound pressure - Precision methods for anechoic and hemi-anechoic rooms (2003).

[2] ISO 6926: 1999 (JIS Z 8739: 2001), Requirements for the performance and calibration of reference sound sources used for the determination of sound power levels (1999).

[3] M. Vorlander and G. Raabe, "Calibration of reference sound source," Acustica, 81, 247-263 (1995).

[4] T. Fujimori, R. Horiuchi and S. Sato, "Measurement of acoustic center position of LS2P laboratory standard microphones," $J$. Acoust. Soc. Jpn. (J), 58, 579-585 (2002) (in Japanese).

[5] K. Yamada, H. Takahashi and R. Horiuchi, "Influence of reflecting plane having finite surface density on sound power level of reference sound sources calibrated in hemi free-field," Proc. Inter-Noise 2014, 9 pages (2014).

[6] IEC 61094-4: 1995, Measurement microphones - Part 4: Specifications for working standard microphones (1995).

[7] For example, M. P. Norton and D. G. Karczub, Fundamentals of Noise and Vibration Analysis for Engineers (Cambridge University Press, Cambridge, 2003), pp. 232-237. 